\title{
Physics and Modeling of Shock-Wave Dispersion in Heterogeneous Composites
}

\author{
D. Grady
}

Applied Research Associates, 4300 San Mateo Blvd., A-220, Albuquerque, NM 87110, U.S.A

\begin{abstract}
Wave dispersion in heterogeneous solids due to scattering within the microstructure is examined. Underlying physics is explored. A theory based on a quasi-harmonic normal model description of the scattering wave energy is developed and compared with phenomenological nonlinear anelasticity models of wave propagation in heterogeneous media.
\end{abstract}

\section{INTRODUCTION}

Wave propagation in a homogeneous linear elastic continuum is well understood. In contrast wave propagation in a heterogeneous and nonlinear elastic media is considerably more complex. Elastic pulse wave transmission in heterogeneous solids exhibit similarities to wave propagation in dissipative media. Pulse wave shapes will evolve and attenuate, while energy is lost from the pulse as the wave propagates. Nonlinear elastic heterogeneous media can support steady structured shock waves and account for the prerequisite entropy production in the compressive shock process. The underlying cause is related to wave dispersion brought about by scattering within the heterogeneous microstructure.

An approach to modeling dispersive wave propagation in heterogeneous solids brought about by acoustic scattering is pursued in the present study. The underlying physics governing finite-amplitude wave propagation in heterogeneous media is explored. In particular, those aspects of elastic heterogeneous solids are examined which lead to structured steady shock waves. A theoretical formulation based on a quasi-harmonic normal mode representation of the scattered wave energy is pursued. Acoustic waves scattered in a heterogeneous solid during passage of a directed shock wave or transient pulse are, in principal, the same in nature as the lattice waves which characterize the thermal state of a solid. They differ only in the wave frequencies dominating the physics with that of the thermal waves being substantially higher than microstructural acoustic waves. It is found that the theory, under relatively modest assumptions, leads to nonlinear anelastic continuum models of wave propagation. Phenomenologica! continuum models proposed by earlier workers to describe dynamics in heterogeneous solids are examined, and found consistent with, the framework of the present theory.

\section{DISPERSION IN THE SHOCK WAVE EVENT}

Finite amplitude compression waves in nonlinear solids will become steeper as the wave propagates. Wave dispersion can counter the steepening process leading to structured steady shock waves. Heterogeneities within a solid body contribute to wave dispersion and can account for structured steady shock waves. To understand the underling physics responsible for structured steady shock waves in heterogeneous solids consider the following hypothetical situation: The material of concern is a nonlinear thermoelastic heterogeneous solid. Paths of elastic uniaxial strain loading corresponding to isothermal conditions, isentropic conditions and steady shock (Hugoniot) conditions for this solids are identified in Figure 1. When subjected to shock compression through a steady structured shock the continuous stress versus strain compression path experienced by a material element departs from the thermodynamic surface, following a straight Rayleigh line from the state in front of the shock to the compressed thermodynamic state behind the shock. 
The following questions concerning shock compression of this elastic heterogeneous solid are instructive: First, what physically supports the excess nonequilibrium stress (the vertical distance between the Rayleigh line and the equilibrium thermodynamic surface) experienced during the shock transition process? Second, what are the underlying physical mechanisms responsible for the structure of the compression shock wave? Third, how is entropy production accounted for in the elastic shock process?

In pursuing answers to the questions posed consider the aspect of a heterogeneous solid illustrated in Figure 2 by the single inclusion in an otherwise continuous solid. A region of the body including this inclusion compressed quasi-statically would achieve some level of lattice strain energy. That same region compressed by a finite amplitude shock wave, however, as illustrated in Figure 2 would, in

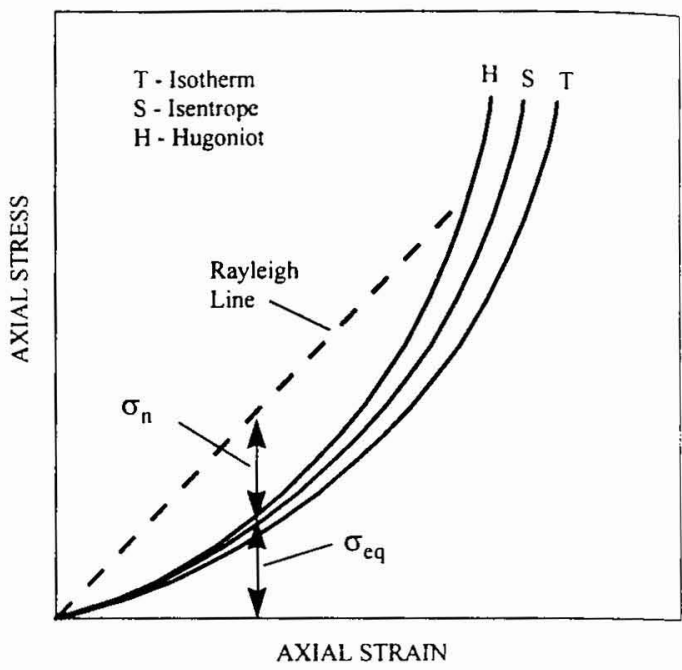

Figure 1: Structured shock process in thermo-elastic solid. addition to the equilibrium lattice strain energy, include a dynamic or acoustic energy component associated with wave scattering as the shock wave passed over the inclusion. In fact, as the characteristic wave length $\lambda$ of the shock approached the characteristic dimension $d$ of the inclusion scattering would be expected to amplify due to near resonance conditions.

Thus, when a finite amplitude shock wave passes through a heterogeneous solid the energy state of the matter behind the shock wave will include a component due to the lattice strain energy and a kinetic component corresponding to a field of acoustic phonon energy caused by wave scattering within the heterogeneous microstructure.

Such phonon energy can be characterized through a quasi-harmonic normal mode representation of an elastic solid. Although more commonly applied in the description of perfect crystals in which: lattice waves and normal modes are the same [1], heterogeneous elastic solids, in principal. can also be described by normal mode coordinates. A property of normal coordinates is a decoupling from the other coordinates such that energy introduced into that coordinate persists. In real solids the elastic constant: depend on distortion (dilation and shear). These anharmonic properties of the material will lead to a coupling of normal mode coordinates and the diffusion of energy throughout the system of normal coordinates until an equilibrium controlled by statistical mechanical principals is achieved. Phonon

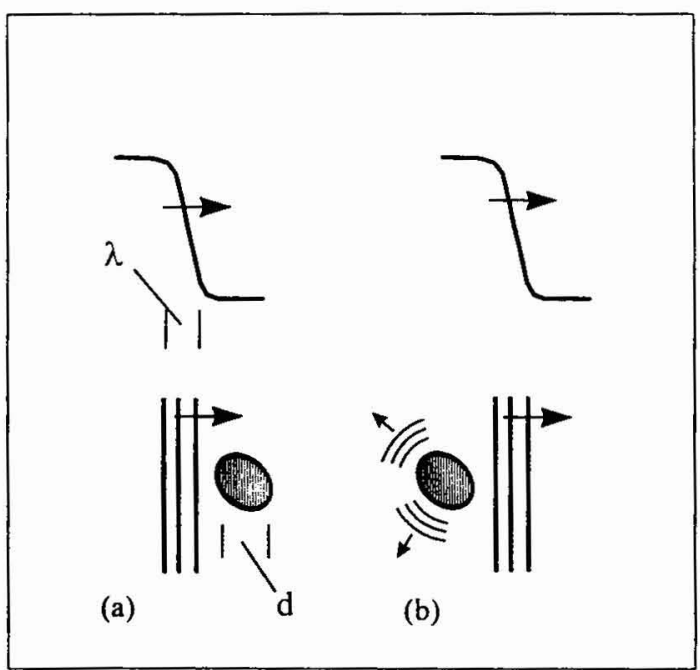

Figure 2: Wave/inclusion interaction resulting in scattering of elastic energy. energy within the system of normal coordinates will also contribute to the global stress state if anharmonicity is a property of the media.

The inducement of a kinetic component of acoustic phonon energy during the dynamics of the shock compression process as outlined in the brie comments above offer answers to the questions posed. During passage of the structured shock dispersion due to wave scattering leads to the production of phonon energy within a limite spectrum of normal mode coordinates in the shoch front. This nonequilibrium acoustic phonon energ! accounts for the excess stress on the Rayleigh lint during passage of the shock wave. Diffusion of this energy to an equilibrium configuration throughou the acoustic normal modes is achieved as the final shock state is approached. Ultimately continued diffusion throughout the full thermal spectrun occurs until complete thermo-elastic equilibrium is achieved. The latter accounts for entrop! production within the elastic shock process in the heterogeneous solid. Details of the steady shoch 
wave profile structure are determined by characteristic relaxation times associated with specific acoustic energy diffusion processes.

\section{NORMAL MODE DESCRIPTION OF KINETICS IN HETEROGENEOUS SOLIDS}

The foregoing description of large-amplitude wave dispersion resulting from acoustic scattering in heterogeneous solids can be described by a quasi-harmonic model of the shock compression process. As noted, the dynamic state of an elastic heterogeneous solid can be characterized by a normal mode system of coordinates.

Accordingly, consider a decomposition of the energy into that due strictly to lattice compression $U_{0}(\varepsilon)$ and that due to the acoustic phonon energy $U_{k}=\sum n_{i} h v_{i}$,

$$
U=U_{o}(\varepsilon)+\sum_{i} n_{i} h v_{i}
$$

The current nominal axial strain state is $\varepsilon$ while $n_{t}$ is the number of phonons with energy $h v$. The normal mode frequency is $v_{i}$ and $h$ is Planck's constant. Although a quantum representation of the acoustic phonon energy may appear superfluous to what is clearly a classical issue, the approach offers a particularly transparent framework for developing the appropriate relations and has been productively used in other well known theoretical solid state concepts such as the Debye theory of specific heat and Gruneisen's theory of thermal pressure.

To proceed $U_{k}$ in Equation 1 will be decomposed into acoustic and thermal normal modes,

$$
U=U_{0}(\varepsilon)+\sum_{i}^{\text {aconssic }} n_{i} h v_{1}+\sum_{i}^{\text {ihcrnal }} n_{i} h v_{i},
$$

where the acoustic-to-thermal transition in vibrational frequency will be of the order $10^{10}-10^{11}$ hertz while the upper cutoff is of the order of the Debye frequency $\left(\sim 10^{15}\right.$ hertz). In equilibrium each normal coordinate would have an energy of $u_{i}=k T$ where $T$ is the temperature. Because the density of vibrational frequencies increases like $v_{i}^{2}$, energies in the acoustic modes under equilibrium conditions are ordinarily negligible. In the present application, however, where energy is initially introduced into a limited spectrum of acoustic modes through scattering within the heterogeneous microstructure, the acoustic portion of the normal mode spectrum is critical to the physical processes. Equilibration throughout the acoustic spectrum and ultimately over the full thermal spectrum can be abstractly depicted in the following way;

$$
U=U_{0}(\varepsilon)+\sum_{i}^{\tau_{a}} n_{i} h v_{i}+\sum_{i}^{\substack{\tau_{1} \\ \text { acomsic }}} n_{i} h v_{i},
$$

where $\tau_{a}$ and $\tau_{\text {, }}$ represent relaxation times over acoustic modes and over the thermal spectrum, respectively. The processes identify a diffusion of phonons into the under populated acoustic modes and the higher frequency thermal modes. in the form,

Recognizing the focus on the acoustic normal mode spectrum we will return to Equation 1 written

$$
U=U_{n}(\varepsilon)+\sum_{i} n_{i v} h v_{1}+\sum_{i}\left(n_{i}-n_{i v}\right) h v_{i},
$$

where $n_{i o}$ represents the set of equilibrium population numbers. Combining the first two terms on the right as the equilibrium thermoelastic equation of state $U_{e q}(\varepsilon, s)$ of the heterogeneous solid gives,

$$
U=U_{e q}(\varepsilon, s)+\sum_{i}\left(n_{i}-n_{i u}\right) h v_{i} .
$$

The second term on the right in Equation 5 is then the nonequilibrium phonon energy induced in the shock-wave scattering process. The strain derivative of $U$ provides the stress,

$$
\sigma=\sigma_{c \psi}+\sum_{i}\left(n_{i}-n_{i}\right) h v_{i}^{\prime},
$$

also composed of an equilibrium component and a contribution due to the nonequilibrium phonon energy. 
The anharmonic character of the respective normal modes is determined by the strain dependence of the normal mode vibration frequencies $v_{i}^{\prime}$. The time dependence of Equation 6 yields,

$$
\dot{\sigma}=\dot{\sigma}_{e q}+\sum_{i} \frac{d n_{i}}{d t} h v_{i}^{\prime} \text {. }
$$

The second term on the right will be identified as $\dot{\sigma}_{n}$ the time rate of the nonequilibrium component of the stress due to the acoustic phonon field stimulated in the heterogeneous solid. Ignored in the step leading to Equation 7 is a nonzero time-dependent term resulting from the strain dependence of the normal mode frequency. Assumed small in the present derivation this term could alternatively be included in the $S(\cdot)$ term defined in Equation 10 below.

The expression $d n_{i} / d t$ is fundamental in modeling the rate of stimulation and relaxation of the phonon population within the acoustic normal modes. A reasonable governing equation would be,

$$
\frac{d n_{i}}{d t}=s_{i}(\cdot)-\frac{1}{\tau_{i}}\left(n_{i}-n_{i v}\right),
$$

where $s_{i}(\cdot)$ represents a phonon stimulation function dependent on the dynamics of the wave interaction with the heterogeneous microstructure. The second term provides for relaxation of the normal mode energy levels toward an equilibrium population $n_{i,}$ with a characteristic relaxation time $\tau_{i}$ for each normal mode. Equation 7 and 8 combine to provide,

$$
\dot{\sigma}=\dot{\sigma}_{e q}+\sum_{i} s_{i} h v_{i}^{\prime}-\sum_{i} \frac{1}{\tau_{i}}\left(n_{i}-n_{i o}\right) h v_{i}^{\prime} .
$$

If one assumes that a common relaxation time $\tau \approx \tau$, sensibly describes all normal modes Equation 9 . with Equation 6, yields,

$$
\dot{\sigma}=\dot{\sigma}_{\iota \varphi}+S(\cdot)-\frac{1}{\tau}\left(\sigma-\sigma_{e q}\right) .
$$

Equation 10 is recognized as the functional form for a continuum nonlinear anelastic constitutive relation and has resulted from a quasi-harmonic description of dispersive wave scattering in heterogeneous media. In the following section phenomenological constitutive models of the form of Equation 10 proposed by earlier authors to describe dispersive wave propagation in heterogeneous media are examined.

\section{MODELING DISPERSIVE WAVE PROPAGATION IN THE HETEROGENEOUS SOLID}

The physical issues of nonlinear large-amplitude wave dispersion introduced in the earlier sections clearly suggest a material response model initiating with the decomposition of stress,

$$
\sigma=\sigma_{e q}+\sigma_{n} \text {. }
$$

This decomposition is not an assumption. It is a recognition that on the most fundamental level forces of interaction in condensed matter are a composition of lattice potential and kinetic (momentun exchange) terms. The first term on the right will determine the equilibrium thermoelastic response of the heterogeneous solid. That this term may also include the equilibrium component of the stress. as suggested by Equations 4 and 5, is not inconsistent with the fundamental stress decomposition of Equation 11. The second term on the right accounts for stresses brought about by the nonequilibrium acoustic phonon energy induced in the heterogeneous microstructure by the transient wave. The assumed time dependence is of the general form,

$$
\dot{\sigma}_{n}=S+R,
$$

where the terms $S$ and $R$ account, respectively, for the stimulation and relaxation of nonequilibrium phonon energy. Within these terms lies the opportunity to model a rich variety of responses exhibited hy heterogeneous solids subjected to finite-amplitude transient stress wave propagation. The general modil framework identified by Equations 11 and 12 and the specific models to be considered presently which fall within this framework will be recognized as continuum anelastic representations of the stress versuls strain response of solid matter. 
Knopoff [2] was perhaps one of the first to recognize the potential of continuum anelasticity models to describe wave dispersion brought about by scattering in heterogeneous media. He realized that heterogeneities within the earth's interior contributed to the dispersion and attenuation of sonic waves and explored the applicability of a linear Maxwell model to describe the wave propagation. The linear Maxwell model within the framework of Equations 11 and 12 is written,

$$
\begin{aligned}
& \dot{\sigma}=\dot{\sigma}_{n}=M \dot{\varepsilon}-\frac{1}{\tau} \sigma_{n}, \\
& \dot{\sigma}_{e y}=0 .
\end{aligned}
$$

In this most fundamental of anelasticity models transient stresses are stimulated through a modulus $M$ and relax over a characteristic time $\tau$. Knopoff found that dispersion characteristics predicted by Equation 13 were not in accord with observed attenuation of sound waves in the earth.

Barker [3] proposed use of a general nonlinear Maxwell model in the form,

$$
\dot{\sigma}-\varphi(\varepsilon) \dot{\varepsilon}=-\frac{1}{\tau}\left(\sigma-\sigma_{e q}\right),
$$

to describe finite amplitude wave propagation in composite solids. This method compared favorably with both experimental data and computer solutions of wave propagation in composites and has been examined in some detail recently by Johnson et al. [4]. The stress decomposition of Equations 11 and 12 is readily produced,

$$
\begin{aligned}
& \dot{\sigma}_{n}=M(\varepsilon) \dot{\varepsilon}-\frac{1}{\tau} \sigma_{n}, \\
& \dot{\sigma}_{e^{\prime} q}=\vartheta(\varepsilon) \dot{\varepsilon} .
\end{aligned}
$$

Equation 16 explicitly displays the stimulation and relaxation terms identified in Equation 12. Stimulation of nonequilibrium stress is accomplished through the modulus $M(\varepsilon)=\varphi(\varepsilon)-\vartheta(\varepsilon)$. Relaxation is governed by a linear relation proportional to the nonequilibrium stress.

Barker's relation nicely models a theory due to Zel'dovich [5] on shock waves in relaxing media in which the normal mode coordinates are considered to be divided into two categories: those which are excited instantly and those which are stimulated only after some characteristic relaxation time. The modulus $M$ then determines the difference in compression paths of the media under the conditions of restricted (nonequilibrium) and unrestricted (equilibrium) stimulation of the normal mode degrees of freedom.

More recently Kanel' et al. [6] have proposed another continuum anelasticity model to describe wave propagation in solid composites. In the same framework the model can be written,

$$
\begin{aligned}
& \dot{\sigma}_{n}=k \dot{\varepsilon}^{m}-\frac{1}{\tau} \sigma_{n}, \\
& \dot{\sigma}_{e q}=\vartheta(\varepsilon) \dot{\varepsilon} .
\end{aligned}
$$

Although quite similar to Barker's model (the models differ only in the stimulation term) the physical implications in the stimulation term for $m \neq 1$ are profoundly different. Rather than modeling a dichotomous (instantaneous and equilibrium) response of the material, the stimulation term implies a continuously increasing scattered energy (and hence nonequilibrium stress) with increasing strain rate within the shock wave. The modeled behavior can be likened, for example, to the Rayleigh scattering of sound waves in heterogeneous media -- an effect which has been observed experimentally in the acoustic limit by Mason and McSkimin [7].

To further emphasize the modeling opportunities offered through the present formulation it is interesting to speculate further on the implications of Rayleigh scattering to wave dispersion in heterogeneous media. For media with a pronounced microscale it is reasonable to expect a resonance in scattering for characteristic wavelengths near this microscale. The theory of Rayleigh scattering leads to a scattering cross-section which might suggest the following term for the stimulation nonequilibrium stress, 


$$
S=\frac{k \dot{\varepsilon}^{4}}{\left(\dot{\varepsilon}-\dot{\varepsilon}_{0}\right)^{2}-\gamma^{2} \dot{\varepsilon}^{2}} .
$$

Such a term resembles that proposed by Kanel' et al. [6] but leads to strong resonance scattering at shock rise-times commensurate with $\dot{\varepsilon}_{0}$.

\section{CONCLUSIONS}

The physics underlying transient wave propagation, in which scattering of wave energy brought about by interaction of the wave with the heterogeneous substructure of a solid, has been explored in the present study. This feature of heterogeneous matter can lead to wave dispersion, wave attenuation and steady. structured waves not unlike wave propagation in dissipative media. A decomposition of energy into potential strain energy and vibrational energy was pursued where a normal mode quasi-harmonic representation of the latter is assumed. Key to the theoretical development is a recognition that transient wave interaction with the microstructure will lead to an initial highly nonequilibrium distribution of the vibrational energy. Statistical mechanical forces will drive the system toward an equilibrium distribution throughout the normal mode spectrum of the body. In application this energy will ultimately thermalize if it is not absorbed by intervening dissipative processes (i.e., damage, dislocation motions) as it percolates through the substructure degrees of freedom.

Relatively general assumptions in modeling the wave stimulation of the vibrational spectrum and the subsequent time-dependent relaxation toward equilibrium is found to lead to nonlinear continum anelastic equations. Phenomenological equations developed and used by earlier workers [2.3.6] to describe the wave propagation in heterogeneous matter are shown to be in accord with the theoretical results. Consequently, a firmer physical basis for such models has been established. More importantly the theoretical methodology points toward a path whereby the dynamics of more complex substructure physical processes can be modeled.

\section{References}

[1] Klemans, P. G., Effects of Thermal and Phonon Process in Ultrasonic Attenuation, Physical Acoustics-IIIB, W. P. Mason Ed. (Academic Press, New York, 1965) pp. 201-234.

[2] Knopoff, L., Attenuation of Elastic Waves in the Earth, Physical Acoustics-IIIB, W. P. Mason Ed. (Academic Press, New York, 1965) pp. 287-324.

[3] Barker, L. M, J. Composite Mat., 5, (1971) 140-162.

[4] Zel'dovich, Ya. B., Zh. Eksperim. i Teor. Fiz., 16, (1946) 365-368.

[5] Johnson, J. N., R. S. Hixson and G. T. Gray, J. Appl. Phys., 76, (1994) 5706-5718.

[6] Kanel', G. I., M.F. Ivanov and A. N. Parshikov, In. J. Impact Engng., 17, (1995) 455-464.

[7] Mason, W. P. and H. J. McSkimin, J. Acous. Soc. Am., 19, (1947) 464-473. 